\title{
Quantitative proteomics analysis of differentially expressed proteins induced by astragaloside IV in cervical cancer cell invasion
}

\author{
Chenglai Xia ${ }^{1 *}$, Zhihong $\mathrm{He}^{1}$ and Yantao $\mathrm{Cai}^{2}$
}

\footnotetext{
*Correspondence: xiachenglai@126. com

${ }^{1}$ Foshan Maternal and Child Health Research Institute, South Medical University Affiliated Maternal \& Child Health Hospital of Foshan, 11 Renmin Xi Street, Foshan 528000, China

Full list of author information is available at the end of the article
}

\begin{abstract}
Background: Cervical cancer remains the second leading cause of mortality in women in developing countries. While surgery, chemotherapy, radiotherapy, and vaccine therapy are being applied for its treatment, individually or in combination, the survival rate in advanced cervical cancer patients is still very low. Traditional Chinese medicine has been found to be effective in the treatment of cervical cancer. Astragaloside IV (AS-IV), a compound belonging to Astragalus polysaccharides, shows anticancer activity through several cell signaling pathways. However, the detailed molecular mechanism governing the anticancer activity of AS-IV remains unknown.
\end{abstract}

Material and methods: In our study, we performed tumor xenograft analysis, transwell cell migration and invasion assay, Western blot analysis, and iTRAQ combination by parallel reaction monitoring (PRM) analysis to study the molecular mechanism of AS-IV in the suppression of cervical cancer cell invasion.

Results: Our results showed that AS-IV suppressed cervical cancer cell invasion and induced autophagy in them, with the tumor growth curve increasing slowly. We also identified 32 proteins that were differentially expressed in the SiHa cells when treated with AS-IV, with 16 of them involved in the upregulation and 16 in the downregulation of these cells. These differentially expressed proteins, which were predominantly actinmyosin complexes, controlled cell proliferation and cell development by steroid binding and altering the composition of the cell cytoskeleton. DCP1A and TMSB4X, the two proteins regulating autophagy, increased in cervical cancer cells when treated with AS-IV.

Conclusions: We conclude that AS-IV could inhibit cervical cancer invasion by inducing autophagy in cervical cancer cells. Since iTRAQ combination by PRM has been observed to be useful in identifying macromolecular target compounds, it may be considered as a novel strategy in the screening of anticancer compounds used in the treatment of cervical cancer.

Keywords: Astragaloside IV, Quantitative proteomics, iTRAQ, Parallel reaction monitoring, Cervical cancer

(c) The Author(s). 2020 Open Access This article is licensed under a Creative Commons Attribution 4.0 International License, which permits use, sharing, adaptation, distribution and reproduction in any medium or format, as long as you give appropriate credit to the original author(s) and the source, provide a link to the Creative Commons licence, and indicate if changes were made. The images or other third party material in this article are included in the article's Creative Commons licence, unless indicated otherwise in a credit line to the material. If material is not included in the article's Creative Commons licence and your intended use is not permitted by statutory regulation or exceeds the permitted use, you will need to obtain permission directly from the copyright holder. To view a copy of this licence, visit http://creativecommons.org/licenses/by/4.0/. 


\section{Introduction}

The cancer data for 2019 reveal that cervical cancer continues to be the second leading cause of mortality in women, particularly in those between 20 and 39 years of age [1]. About nine cancer patients are observed to die from cervical cancer every week [2]. While surgery, chemotherapy, radiotherapy, and vaccine therapy are applied for its treatment, individually or in combination, the survival rate in advanced cervical cancer patients is still very low, and the side effects of the therapies are continuing to increase [3, 4]. In addition, the 2-, 4-, and 9-valent HPV vaccines do not show any clinical effectiveness in the treatment of cervical cancer [5-7]. Therefore, there is an urgent need to explore more effective drugs to prevent cervical cancer cell invasion and improve the quality of life in cervical cancer patients.

Several anticancer drugs have been derived from natural products [8]. There are about 350,000 plant species on earth, which have been a major source of medicines for a long time. The Chinese began to use herbs to treat cancer, as well as cardiovascular and other incurable diseases, several thousands of years ago $[9,10]$. Astragalus roots which have been used in traditional Chinese medicine include those of Astragalus mongholicus and $A$. membranaceus [11]. Astragalus has the effect of invigorating qi for strengthening superficies, diuresis, platoon poison, discharging pus and accumulating sore and muscle [12]. Astragaloside IV (AS-IV), a lanolin alcohol-form tetracyclic triterpenoid saponin, is an active ingredient of the Chinese herb Astragalus [13]. Researchers have found that AS-IV showed anticancer effects by targeting the JNK/c-Jun/AP1, Akt/GSK-3 $\beta / \beta$-catenin, PI3K/ Akt/NF-kB, and other signaling pathways [12, 14-17]. However, the detailed molecular mechanism governing the anticancer activity of AS-IV remains unknown. Therefore, in this study, iTRAQ proteomics was used to identify the differentially expressed proteins in cervical cancer cells treated by AS-IV and aim to discover in detail the molecular mechanism governing the anticancer activity of AS-IV.

\section{Methods and materials}

Cells, antibodies and siRNA

SiHa and HeLa cells, two kinds of human cervical cancer cell lines, were obtained from Dr. Xia's laboratory (Foshan, China). Fetal bovine serum was bought from Thermo Fisher Scientific (Carlsbad, CA, USA). AS-IV (molecular weight, 784.97) was obtained from Sigma-Aldrich Corporation (St Louis, MO, USA). The antibodies rabbit antihuman LC3I/II, GAPDH, Atg7, and Atg12, and their corresponding secondary antibodies were acquired from Cell Signaling Technology Inc. (Beverly, MA, USA). DCP1A siRNA and TXSB4X siRNA were purchased from Sangon Biotech Co., Ltd. (Shanghai, China).

DCP1A siRNA sequence: sense (5'-3'), GACACAACCACUUGGGAAATT; antisense (5'-3'), UUUCCCAAGUGGUUGUGUCTT.

TXSB4X siRNA sequence: sense (5'-3'), AGACAGAGACGCAAGAGAATT; antisense (5'-3'), UUCUCUUGCGUCUCUGUCUTT.

\section{Cell culture}

The SiHa and HeLa cells were cultured in Dulbecco's modified eagle medium (DMEM) containing $10 \%$ fetal bovine serum (Invitrogen, Carlsbad, CA, USA) and $1 \%$ 
streptomycin (Sigma-Aldrich, St. Louis, MO, USA). Cells $\left(1 \times 10^{7}\right)$ were harvested by trypsinization after treatment with $25 \mu \mathrm{M}$ astragaloside IV or not for $12 \mathrm{~h}$ and washed 5 times by using of PBS.

\section{Western blot analysis}

Based on our previous study [18], cell total protein samples were taken from a $-80{ }^{\circ} \mathrm{C}$ refrigerator. After the addition of lysis buffer, the samples were centrifuged at $12,000 \mathrm{~g}$ at $4{ }^{\circ} \mathrm{C}$ for $10 \mathrm{~min}$. The supernatants were transferred to new centrifuge tubes and quantified using a BCA kit (Novagen, MA, USA). The precooled protein samples and predyed markers were added to the gel. After running the gel, protein was transferred onto the PVDF membrane (Millipore, MA, USA). The non-specific antigens on the PVDF membrane surface were blocked by $5 \%$ non-fat milk. After hybridization with the corresponding primary and secondary antibodies and washing with Tris-buffered saline $(0.1 \%$ Tween 20$)$, the PVDF membrane was developed using an enhanced chemiluminescence solution (Pierce, IL, USA) and subsequently photographed using a BioRad gel imaging system.

\section{Cancer cell invasion analysis}

Based on our previous study [18], 24-well transwell invasion chambers (Corning, NY, USA) were used to analyze the cancer cell invasion. The matrix gel $(1 \mathrm{mg} / \mathrm{ml}$; Bio-Rad, CA, USA) was introduced into the upper chamber and the cell culture medium containing $10 \%$ fetal bovine serum was introduced into the lower chamber; $2 \times 10^{5}$ perwell HeLa or SiHa cells were seeded into the upper chamber. After treatment with ASIV for $12 \mathrm{~h}$, the cells that had invaded the lower chamber were fixed with methanol and dyed using crystal violet. We used an inverted microscope to count the number of invaded cells.

\section{iTRAQ proteomics}

Based on a previous study [19], the labeling agent of the iTRAQ (Thermo, USA) kit was dissolved in acetonitrile buffer after thawing. Briefly, cell samples were harvested in lysis buffer ( $8 \mathrm{M}$ urea and $1 \%$ protease inhibitor cocktail) and sonicated three times on ice with a high-intensity ultrasonic processor (Scientz, China). The protein supernatant concentration was measured with a BCA kit after centrifugation at $12,000 \mathrm{~g}$ at $4{ }^{\circ} \mathrm{C}$ for $10 \mathrm{~min}$ according to the manufacturer's instructions. Three hundred micrograms of protein in pre-samples was subjected to analysis. The peptide was lysed with trypsin and desalted with Strata X C18, drying in a vacuum container. The dried peptide was labeled by mixing with a labeling agent for $2 \mathrm{~h}$ at room temperature, desalted, and lyophilized in a vacuum container. Then, the tryptic peptides were fractionated using a C18 column with $5 \mu \mathrm{m}$ particles, $4.6 \mathrm{~mm}$ ID and $250 \mathrm{~mm}$ length (Thermo Betasil) equipped in a high $\mathrm{pH}$ reverse-phase HPLC machine.

The tryptic peptides were dissolved with $0.1 \%$ formic acid and were subjected to an nanospray ionization (NSI) source followed by tandem mass spectrometry (MS/MS) in Orbitrap Fusion Lumos (Thermo, USA) coupled online to the UPLC. $2.0 \mathrm{kV}$ of electrospray voltage was applied and 350 to 1550 of $\mathrm{m} / \mathrm{z}$ scan range was applied for a full system scan. After being detected at a resolution of 60,000 or 15,000 in the Orbitrap, 
intact peptides or fragments were selected for MS/MS using the NCE setting of 28 . The data-dependent procedure alternated between one MS scan followed by $20 \mathrm{MS} /$ MS scans with $30.0 \mathrm{~s}$ dynamic exclusion. Automatic gain control (AGC) was set at 5E4. Fixed first mass was set as $100 \mathrm{~m} / \mathrm{z}$. The resulting MS/MS data were processed using the Maxquant search engine (v.1.5.2.8). In a brief, tandem mass spectra were searched against the human UniProt database (Human_SwissProt_1808) concatenated with the reverse decoy database. Allowing up to 2 missing cleavages, trypsin/P was considered as the cleavage enzyme. The mass tolerance for precursor ions was set as $20 \mathrm{ppm}$ in first search and was set as $5 \mathrm{ppm}$ in the main search. $0.02 \mathrm{Da}$ was set as the mass tolerance for fragment ions. FDR was adjusted to less than $1 \%$ and the minimum score for modified peptides was set to more than 40 . The peptide was identified by its secondorder spectrum and quantified by its intensity. FDR was adjusted to less than $1 \%$ and the minimum score for modified peptides was set to more 40. The mass error (ppm) needed to be within \pm 10 .

\section{Protein function enrichment and cluster analysis}

Based on a previous study [20], collecting the protein enrichment information classified with different functions and corresponding $P$-values, we identified a significant enrichment functional classification $(P<0.05)$. At first the screened $P$-value data matrix was logarithmically transformed $(-\log 10$ model), then the transformed data matrix was used to classify the functions by $\mathrm{Z}$ transform, and finally the hierarchical clustering (Euclidean distance, average connection clustering) method was used for unilateral clustering analysis of the dataset obtained after $\mathrm{Z}$ transformation. A heatmap with the $\mathrm{R}$ language package gplots was drawn to show the cluster relation.

\section{Protein-protein interaction and network analysis}

Based on a previous study [20], differentially expressed proteins screened from different comparison groups were numbered and the protein-protein interactions were identified using the STRING (v.10.5) protein network database. Based on the confidence scores (a score of 0.7 was considered as a high confidence score), the differential protein interactions were extracted and the corresponding protein-protein interaction networks were visualized using the network D3 package.

\section{PRM analysis}

Based on the method mentioned by Sun BB [21], dithiothreitol and iodide acetamide were added to the protein solution. The mixture was hydrolyzed overnight at $37^{\circ} \mathrm{C}$ and the ratio of trypsin:protein was 1:50. The hydrolysis was continued for $4 \mathrm{~h}$ and the ratio of trypsin:protein was 1:100. The peptides were dissolved in $0.1 \%(\mathrm{v} / \mathrm{v})$ aqueous formic acid, and the mobile phase was isolated using the EASY-nLC1000 high-performance liquid chromatography system (Thermo Scientific, Carlsbad, CA). The isolated peptides were subjected to an NSI source followed by mass spectrometry using the Q Exactive Plus Hybrid Quadrupole-Orbitrap (Thermo Scientific) mass spectrometer. The tandem mass spectrometry (MS/MS) data were processed using Skyline (v.3.6). Full details are given in Supplementary Methods. 


\section{Animal experiments}

Four-week-old BALB/c nude mice weighing 15-17 g were provided by the Experimental Animal Center of Guangdong Province (SCXK No. SCXK [yue] 2006-0015) and fed in specific pathogen-free animal rooms. All animal experimental protocols were approved by the Medical Ethics Committee of Southern Medical University affiliated Maternal \& Child Health Hospital of Foshan City (Guangdong Province, China). The temperature and the humidity in the feed room were $22-25^{\circ} \mathrm{C}$ and $40-60 \%$, respectively. The light and dark alternating times were $12 \mathrm{~h} / 12 \mathrm{~h}$. A SiHa cell suspension (0.1 $\mathrm{mL}$; approximately $5 \times 10^{6}$ cells) cultured using serum-free medium was injected into the nude mice left fore region near the axilla. Subcutaneous tumor volumes from the mice were measured using an electronic vernier caliper. Mice with tumor diameter $0.3-0.5 \mathrm{~cm}$ were numbered and randomly divided into 3 groups with 5 mice in each group. AS-IV $(25 \mathrm{mg} / \mathrm{kg} / \mathrm{d})$ was gavaged into the mice. The growth data of the tumor were record twice a week and the tumor volume was calculated using the following formula: $\mathrm{V}=0.5 \times \mathrm{A} \times \mathrm{B}^{2}$, where $\mathrm{V}$ is the tumor volume, $\mathrm{A}$ is the longest diameter of the tumor, and $B$ is the shortest diameter of the tumor. The mice were sacrificed and photographed after 35 days. All experimental procedures were approved by the Institutional Animal Care and Use Committee of South Medical University Affiliated Maternal \& Child Health Hospital of Foshan (Foshan, China).

\section{Statistical analysis}

The SPSS16.0 software (IBM SPSS, Chicago, IL, USA) was used for data analysis in this study. Student's t-test and one-way ANOVA followed by Dunnett's test, at $5 \%$ probability level, were applied for data of morphological and mutagenic assessments. The significance of differences was assessed by Student's t-test and one-way analysis of variance (ANOVA) followed by Dunnett's test, and $P$ values less than 0.05 were considered statistically significant.

\section{Results}

Inhibition of cervical cancer cell proliferation, invasion and xenograft tumor growth by AS-IV

Autophagy, a process of phagocytosis of the cytoplasmic proteins or organelles and their inclusion into vesicles, fusion with lysosomes to form autophagy lysosomes and degradation of the contents they contain, can inhibit inflammation caused by cancer cells and stabilize cell chromosome construction [22, 23]. AS-IV, an Astragalus polysaccharide with a distinct structure, is present in Astragalus in smaller amounts when compared with other polysaccharides [24]. We treated cervical cancer cells with AS-IV for $72 \mathrm{~h}$ followed by the analysis of cell proliferation with CCK-8. As shown in Fig. 1a, AS-IV directly inhibited the proliferation of HeLa and SiHa cell lines infected by HPV18 or HPV-16 respectively. The $\mathrm{IC}_{50}$ values of AS-IV for inhibiting cervical cancer cells were $0.49 \pm 0.03 \mathrm{mM}$ and $0.27 \pm 0.03 \mathrm{mM}$ in $\mathrm{HeLa}$ and $\mathrm{SiHa}$ cells, respectively. However, both the antivirus and anticancer effects of AS-IV are higher than those of the other Astragalus polysaccharides [25, 26]. In our study, we used AS-IV $(12.5 \mathrm{mg} / \mathrm{kg} / \mathrm{d}$, $25 \mathrm{mg} / \mathrm{kg} / \mathrm{d}, 50 \mathrm{mg} / \mathrm{kg} / \mathrm{d}$ ) to treat human cervical cancer xenografts in mice, and chose cisplatin as a positive drug to treat cancer. After 35 days of treatment, the tumor 


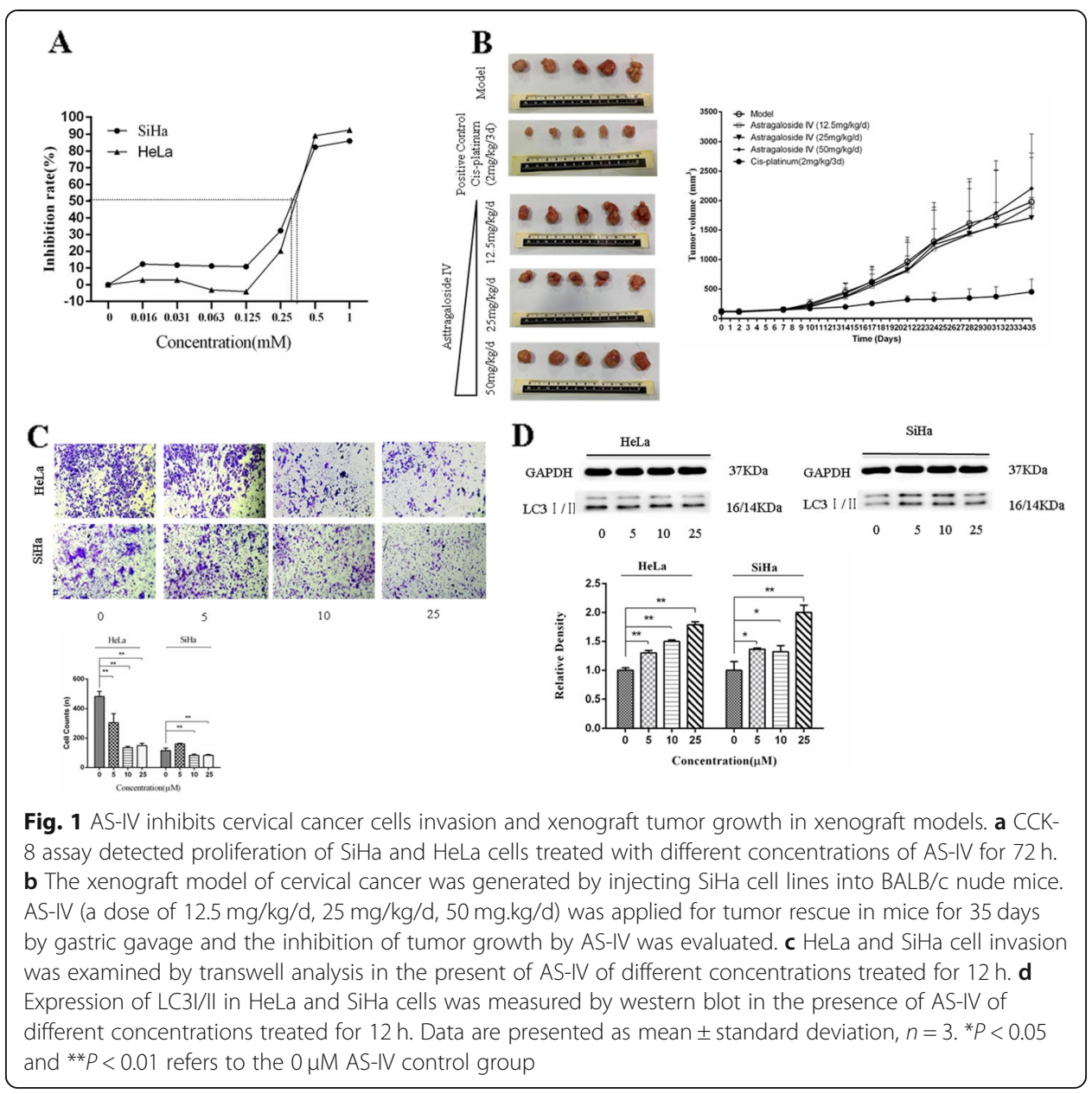

volume in the cisplatin treatment group had obviously shrunk when compared to that in the PBS-treatment group (Fig. 1b). It is, therefore, inferred that our anticancer drug treatment system was effective in the treatment of cervical cancer. Moreover, the tumor volume in the AS-IV $(25 \mathrm{mg} / \mathrm{kg} / \mathrm{d})$ treatment group decreased compared to the PBStreatment group. We used AS-IV $(0,5,10,25 \mu \mathrm{M})$ to treat HeLa and SiHa cells. After $12 \mathrm{~h}$ of treatment with AS-IV, we found that the cancer cell invasion was inhibited in the HeLa and SiHa cells (Fig. 1c). LC3I/II, a protein marker of autophagy, was induced in the HeLa and SiHa cells (Fig. 1d). These results showed that AS-IV inhibited the growth of cervical cancer cells, especially inhibiting the growth of HPV-16-infected human cervical cells, and inhibited xenograft tumor growth by inducing autophagy.

\section{Analysis of differentially expressed proteins by iTRAQ}

In order to understand in detail the mechanism of cell autophagy induced by AS-IV to suppress inflammation in the progression of cervical cancer, we analyzed the differential protein expression in AS-IV-treated cervical cancer cells using the iTRAQ combination. Peptides digested by trypsin were analyzed using high-resolution mass spectrometry (Fusion Lumos). The primary ions and secondary fragments of the peptides were also analyzed using high-resolution mass spectrometry (Fusion Lumos). All 
obtained data were analyzed through a certain bioinformatics database (Fig. 2a). Most of the peptide fragments were found to contain 7-20 amino acid residues, an observation that conforms with the general rules of trypsin enzymatic hydrolysis and HCD fragmentation (Fig. 2b). There was a negative correlation between molecular weight and the protein sequence coverage. Theoretically, larger molecular weight proteins can produce more enzymatic fragments to achieve the same coverage. That is to say, it needs more peptides identified from a large protein to achieve the same coverage. The length of the peptide fragments identified meets the quality control requirements (Fig. 2c). In this experiment, we identified 6964 proteins, of which 6072 proteins were quantified. Considering 1.2-fold expression as the standard we found that 32 proteins were differentially expressed when the SiHa cells were treated with $25 \mu \mathrm{M}$ AS-IV for $12 \mathrm{~h}$. Out of these 32 differentially expressed proteins, the expression of 16 proteins was upregulated and that of the other 16 was downregulated (Fig. 2d).

\section{Functional classification of differentially expressed proteins in AS-IV-suppressed cervical cancer cells}

Cluster analysis, based on the functional enrichment of the differentially expressed proteins, could analyze the potent relationship or distinction between the proteins based on the Gene Ontology (GO) Database, Kyoto Encyclopedia of Genes and Genomes (KEGG) pathway, and their protein domains [25, 27]. GO is an important bioinformatics tool for analyzing the attributes of genes and genetic products, including their biological processes (BPs), cellular components (CCs), and molecular functions (MFs). In our study, we analyzed the distribution of differentially expressed proteins in the secondary annotation of GO. As shown in Fig. 3a, most differentially expressed proteins following the treatment with AS-IV to suppress cervical cancer invasion take part in BPs such as cellular (18\%) and single-organism (26\%) processes, biological regulation (12\%), metabolic process (12\%), and response to stimuli (8\%). Similarly, differentially

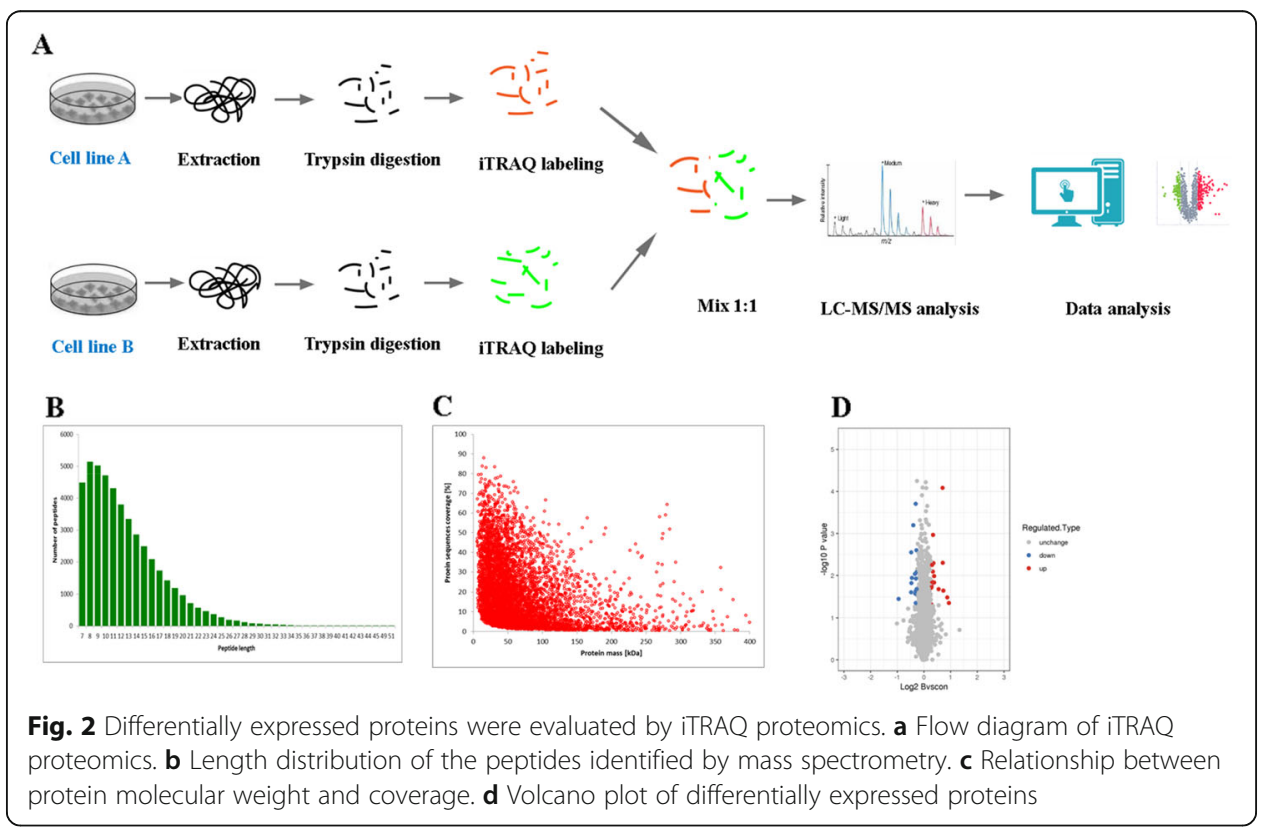



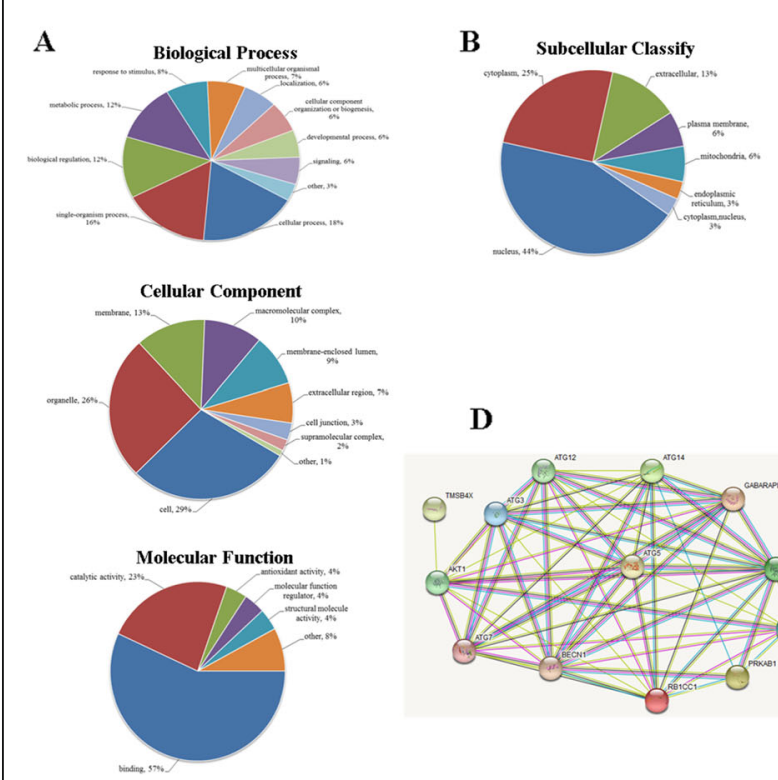

C

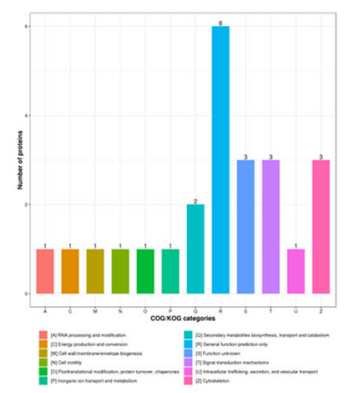

D

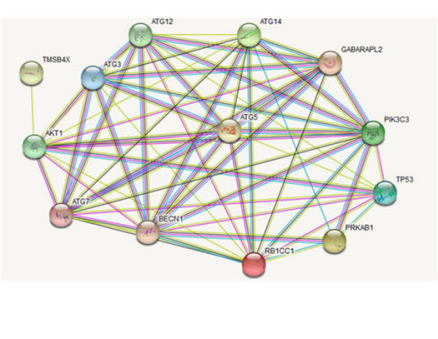

$\mathbf{E}$

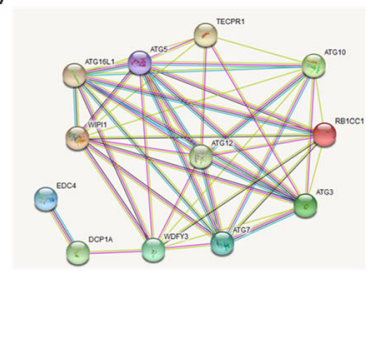

Fig. 3 Differentially expressed proteins under the presence of AS-IV treatment in cervical cancer cells analyzed by Gene Ontology. a BPs, CCs, and MFs evaluated. b Subcellular localization and classification of differentially expressed proteins. c Clusters of orthologous group classification of differentially expressed proteins. d TMSB4X/Akt/Atg5/Atg12 pathway; e DCP1A/WDFY3/Atg12 pathway. Proteins are represented by colored spheres, and interaction proteins are represented by white spheres. The interactions already recorded in the database are shown with blue lines and the interactions that are confirmed by our results are shown in purple. Two adjacent genes are linked by green lines and gene fusion is shown with red lines

expressed proteins following the treatment with AS-IV to suppress cervical cancer invasion belong to cell (29\%), organelle (26\%), membrane (13\%), and macromolecular (10\%) complexes as part of CCs (Fig. 3a). As shown in Fig. 3a in MFs, differentially expressed proteins following the treatment with AS-IV to suppress cervical cancer invasion exhibit MFs such as binding activity (57\%), catalytic activity (23\%), antioxidant activity (4\%), and MF regulator activity (4\%).

InterProScan (http://www.ebi.ac.uk/interpro/) was utilized to analyze the subcellular classification of the differentially expressed proteins. As shown in Fig. 3b, out of the 32 proteins, 8 are located in the cytoplasm, 4 in the extracellular matrix, 14 in the nucleus, 2 in the plasma membrane, 2 in the mitochondria, and one each in the cytoplasm and endoplasmic reticulum. We also analyzed the clusters of orthologous groups of proteins (COG) of these differentially expressed proteins. As shown in Fig. 3c, out of the 32 proteins, there are 3 signal transduction proteins, 1 RNA processing and modification protein, 1 cell wall biogenesis protein, 1 cell motility protein, 3 cytoskeleton proteins, 1 intracellular trafficking, secretion, and vesicular transport protein, 1 molecular chaperone, 1 energy production protein, 1 inorganic ion transport and metabolism protein, 2 secondary metabolite biosynthesis proteins, 6 general function prediction proteins, and 3 unknown-function proteins. Protein-protein interaction network, a useful tool for analyzing key genes, has been applied in many bioinformatics studies [28]. STRING, a database of known and predicted protein-protein interactions, has been widely used in predicting the interactions between known and unknown proteins. As shown in Fig. 3d and e, we found that AS-IV upregulated Atg12 and induced cancer cell autophagy through DCP1A and TMSB4X. The results suggested that the 
progression of AS-IV in the suppression of cervical cancer invasion is related to cell signaling transduction, secondary metabolite biosynthesis, and transmembrane transport, and especially might target DCP1A and TMSB4X.

\section{Functional enrichment of differentially expressed proteins in AS-IV-suppressed cervical cancer cells}

Based on the protein notes, we analyzed the functional enrichment of the differentially expressed proteins at three levels: GO classification, KEGG pathway, and protein domain. We obtained the $P$-value using Fisher's exact test and showed the functional classification or pathway of differentially expressed protein enrichments through a bubble diagram. The vertical axis of the bubble diagram represents the functional classification or pathway and the horizontal axis represents the ratio of differentially expressed proteins to the whole identified proteins, converting from log2. The color of the cycle represents the significant enrichment P-value, and its periphery represents the number of differentially expressed proteins in the pathway. Most of the differentially expressed proteins are involved in BPs such as cell growth and cell development regulation (Fig. 4a), and the CC of a majority of them are actin-myosin complexes (Fig. 4b). Similarly, the differentially expressed proteins perform MFs such as steroid binding, antioxidant activity, and altering the composition of the cell cytoskeleton (Fig. 4c).

The KEGG pathway is used to analyze the interactions among known MFs such as metabolic pathways, formation of complexes, and biochemical reactions. A protein domain, which consists of 25 to 500 amino acid residues, is a repeat sequence occurring during the progression of protein evolution. As may be seen in Fig. 4d, the differentially expressed proteins are enriched in chemical carcinogenesis and the RNA decay signal pathway. As shown in Fig. 4e, the proteins are largely PH domains interacting in nature. The results show that AS-IV regulates several cell-growth proteins, thereby suppressing cervical cancer cell growth.

\section{Induction of autophagy and suppression of cervical cancer invasion by AS-IV by targeting DCP1A and TMSB4X}

PRM analysis, a principal method used to verify the proteomics data, selected specific peptides or target peptides and quantified the target protein or modified the peptides [29]. In the present study, we analyzed 5 of the 32 differentially expressed proteins using PRM instead of the traditional western blot. The results of the iTRAQ analysis showed that the expression of DCP1A and TMSB4X increased, whereas that of MGST3, AKR1C2, and ERL1N1 decreased (Fig. 5a). We observed the same results after the PRM analysis also. The quantitative information of PRM protein is calculated from the ion peak area of the peptide fragment (Supplement Table 1). We also measured the expression of Atg7 and Atg12, the key proteins involved in the autophagy process, in the presence of AS-IV. As shown in Fig. 5b, the expression of Atg7 and Atg12 increased in the presence of AS-IV. Meanwhile, as shown in Fig. 5c, we found that DCP1A siRNA or TMSB4X siRNA could rescue the AS-IV-induced inhibition of cell invasion. We also measured the expression of LC3I/II, Atg7, and Atg12 in the HeLa and SiHa cells. As shown in Fig. 5d, we found that AS-IV could upregulate the expression of LC3I/II, Atg7, and Atg12. However, the expression of LC3I/II, Atg7, and Atg12 


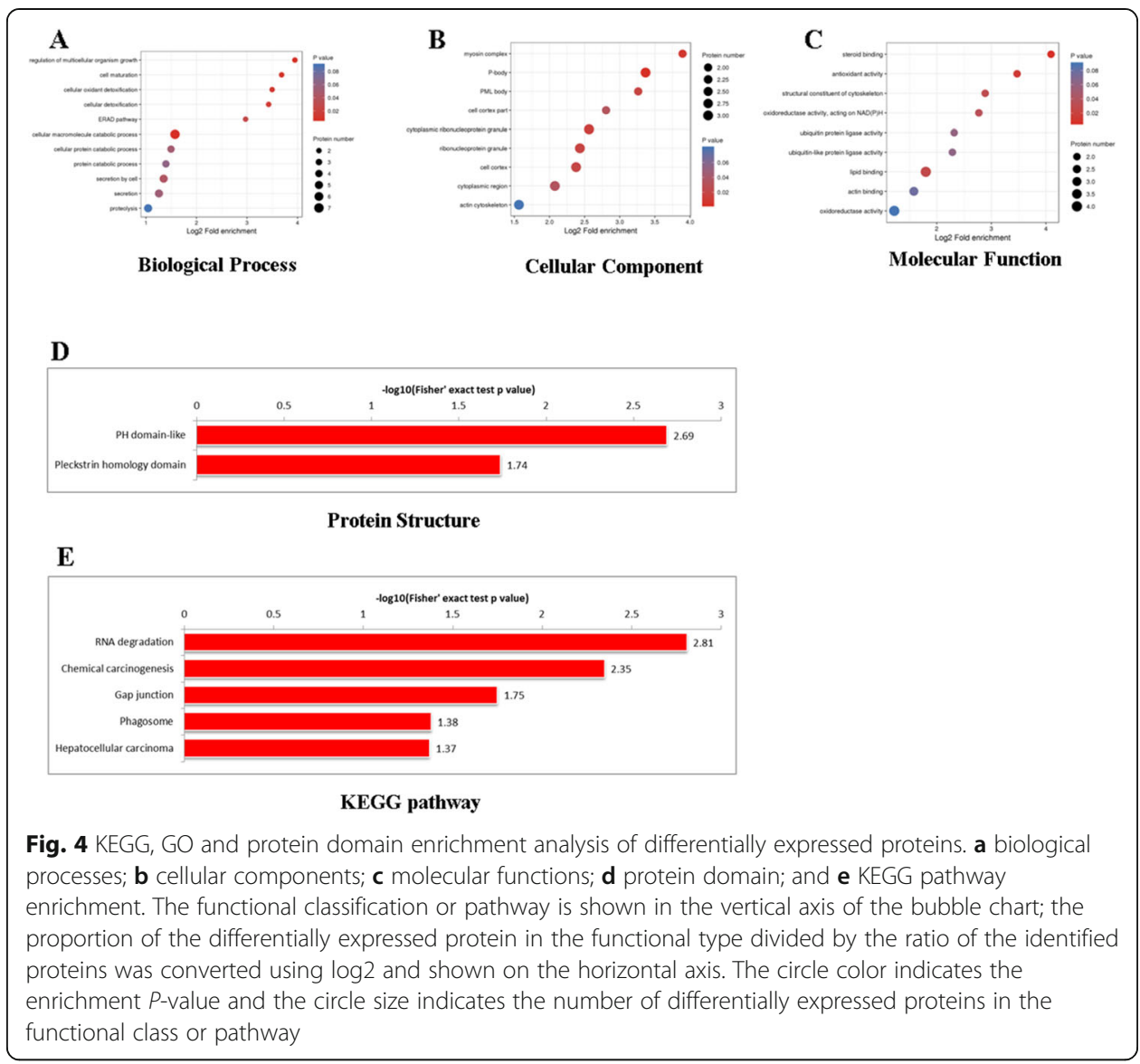

decreased in the presence of AS-IV and DCP1A siRNA or TMSB4X siRNA. We also drew a mechanism map of AS-IV inducing autophagy through the Atg7/Atg12 pathway (Fig. 5e). Thus, DCP1A and TMSB4X may be regarded as the targets of cervical cancer treatment. These results suggest that AS-IV could target the proteins DCP1A and TMSB4X and induce autophagy, resulting in the suppression of cervical cancer proliferation and invasion.

\section{Discussion}

In recent years, the identification of drug targets is the key challenge faced by practitioners in the field of precision pharmacology [30-32]. It is, therefore, necessary to establish a precision pharmacology system. However, the detailed pathological or pharmacological mechanisms that were inferred from related signaling pathways could not completely explain the molecular mechanism governing the anticancer activity of certain drugs [33, 34]. Since the physiological conditions are diverse and the signaling pathways are complex in vivo, several drug side reactions may occur if the drug targets are inaccurate, thereby causing drugs to work less effectively [35]. In the precision medicine and pharmacy era, it is, therefore, beneficial to optimize the physicochemical properties and action targets in terms of spatial and temporal distributions and action intensity as an effort to maximize the specificity of drug effects, reduce uncontrollable side effects, and improve the efficiency of research and development of specific therapeutic drugs for specific indications. During the occurrence and progression of diseases, 


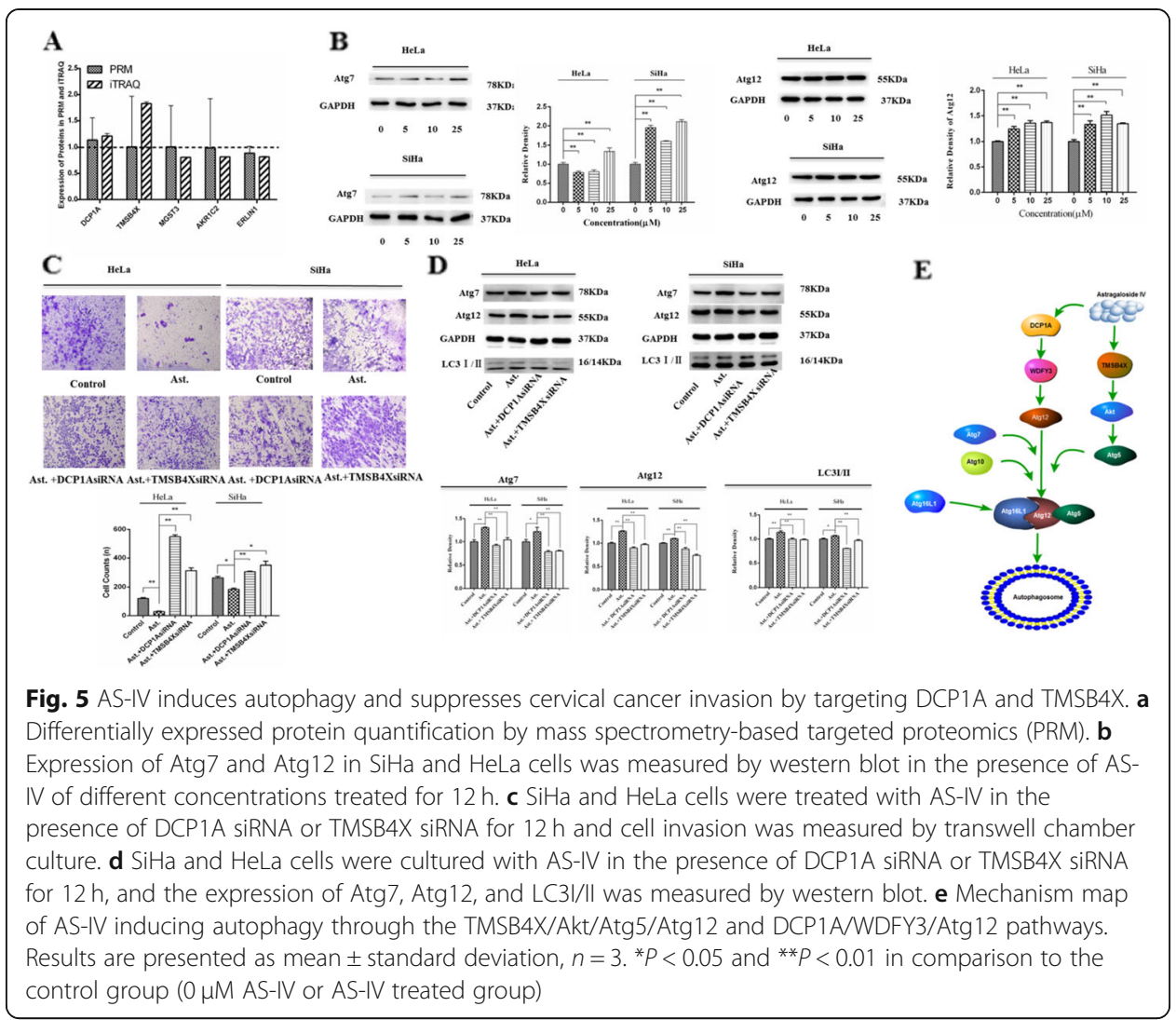

drugs perform their functions mainly by targeting proteins and multiple signaling pathways they participate in. Proteomics, the study pertaining to protein products encoded and expressed by genes, is the way of understanding the interactions of protein molecules in the human body. Proteomics can, thus, reflect the dynamic changes in the physiological and pathological activities in the bodies [36]. The technology is currently being applied to understand several life phenomena that are unable to be explained at the genetic level. The proteomics of cells or bodies under normal conditions, diseases, and during drug treatment helps in the study of the molecular mechanism of the action of macromolecules and the identification of new drug targets, drug development, and clinical treatment guidelines [37]. iTRAQ combination by PRM is applied to identify the protein targets of macromolecules. First, the four-stage bar mass analyzer which has a selective detection capability is used to selectively detect the parent ion information of the target peptide segment. Then the samples are scattered in the HCD collision pool. Finally, all the fragments are transferred into the selected parent ion window and analyzed using the Orbitrap analyzer for high resolution and high accuracy. Thus, the target proteins or peptides in complex samples are accurately analyzed using the PRM system [38]. The technique, a combination of the high selectivity of a four-stage bar, high resolution, and high precision characteristics of the Orbitrap, shows its characteristic advantages including high-resolution sub-ion monitoring, wide second-level full scanning with its linear range up to 5-6 orders of magnitude, and simultaneous qualitative and quantitative analysis [39]. 
AS-IV may be categorized as a macromolecular compound because its molecular weight (784.97) is more than 500. In our study, we performed the iTRAQ combination using PRM to study the potential molecular mechanism of the suppression of cervical cancer cell invasion by AS-IV. We have considered the differential expression multiple of $>1.2$-fold as the criterion for differential protein screening. Our results showed that a total of 32 proteins were differentially expressed, with 16 of them showing increased expression and the other 16 showing decreased expression in the SiHa cells in the presence of AS-IV. It is suggested that AS-IV is different from smaller macromolecular compounds and has no multiple "target holes" in the body. The target of AS-IV may be among these 32 differentially expressed proteins. Subsequently, we assessed the 32 differentially expressed proteins with respect to their BPs, CCs, and MFs. The results showed that the proteins are regulatory proteins controlling cell proliferation and development. While the $\mathrm{CC}$ of these proteins are actin-myosin complexes, they perform MFs such as steroid binding and altering the composition of the cell cytoskeleton. The role of PRM is to validate the proteomic data of iTRAQ, just as using q-PCR to validate transcriptome data. Therefore we selected several key differentially expressed proteins from iTRAQ proteomic data, such as protein DCP1A, TMSB4X, MGST3, AKR1C2, ERLIN1, and then obtained more accurate quantitative values of these proteins by PRM. We found that five of these differentially expressed proteins showed the same expression tendency as that inferred through iTRAQ analysis (Fig. 5a). Our results showed that the protein expression of DCP1A and TMSB4X increased in cervical cancer cells in the presence of AS-IV. Our results also showed that the protein expression of MGST3, AKR1C2, and ERLIN1 decreased in cervical cancer cells in the presence of AS-IV. We also found that AS-IV could target the proteins DCP1A and TMSB4X and induce autophagy in suppressing cervical cancer invasion. The function of the five proteins is related to the progress of tumor cell biology. Out of these five proteins, DCP1A regulates Atg12 and is linked to autophagy and proteasome degradation pathways [40], TMSB4X regulates TGF $\beta 1$ and participates in the progression of cancer [41], MGST3 increases in the LT97 adenoma cells and participates in cancer development [42], AKR1C2 is a key enzyme in the process of progesterone metabolism and is closely related to gynecological tumor development [43], and ERLIN1 is influences the survival time of pancreatic cancer patients [44].

In summary, we performed iTRAQ combination by PRM to identify the potential target of AS-IV in the process of suppressing cervical cancer cell invasion. AS-IV could inhibit cervical cancer invasion by inducing autophagy. While DCP1A and TMSB4X may be regarded as targets of AS-IV in the process of autophagy, they also may be regarded as targets in the screening of anticancer compounds used in the treatment of cervical cancer. Thus, iTRAQ combination by PRM can be used in the identification of macromolecular target compounds and can also be considered as a novel technique in the screening of anticancer compounds used in the treatment of cervical cancer.

\section{Supplementary information}

Supplementary information accompanies this paper at https://doi.org/10.1186/s11658-020-00218-9.

Additional file 1. 


\section{Abbreviations}

AS-IV: Astragaloside IV; iTRAQ: Isobaric tags for relative and absolute quantitation; PRM: Parallel reaction monitoring; DCP1A: mRNA-decapping enzyme 1A; TMSB4X: Thymosin beta-4; MGST3: Microsomal glutathione S-transferase 3; AKR1C2: Aldo-keto reductase family 1 member C2

\section{Acknowledgements}

We thank Jingjie PTM BioLabs (HangZhou, China) for supporting iTRAQ and PRM proteomics analysis.

\section{Authors' contributions}

Study design: $C X$; literature research, data acquisition and data analysis: $C X$, ZH and YC; manuscript preparation and manuscript editing: CX; manuscript review: CX; iTRAQ experiments: $C X$; cell experiments and animal experiments: $Z H$ and $Y C$; final approval of the version to be published: $\mathrm{CX}$, ZH and $\mathrm{YC}$.

\section{Funding}

This study was supported by grants from Foshan Medicine Dengfeng Project (2019-2021), Foshan Anti-Tumor Innovative Drug Research And Engineering Technology Center (FSOAA-KJ819-4901-0082), Medical Project of Science and Technology Department of Foshan (2018AB000031), Science and Technology Bureau of Foshan (FSOAA-KJ218-1301-0008),

\section{Availability of data and materials}

Not applicable.

\section{Ethics approval and consent to participate}

All animals were provided by the Experimental Animal Center of Guangdong Province (SCXK No. SCXK [yue] 20060015). All animal experimental protocols were approved by the Medical Ethics Committee of Southern Medical University affiliated Maternal \& Child Health Hospital of Foshan City (Guangdong Province, China).

\section{Competing interests}

The authors declare that they have no competing interests.

\section{Author details}

${ }^{1}$ Foshan Maternal and Child Health Research Institute, South Medical University Affiliated Maternal \& Child Health Hospital of Foshan, 11 Renmin Xi Street, Foshan 528000, China. ${ }^{2}$ Department of Dermatology and Pheumatology, South Medical University Affiliated Maternal \& Child Health Hospital of Foshan, 11 Renmin Xi Street, Foshan 528000, China

Received: 20 November 2019 Accepted: 17 March 2020

Published online: 31 March 2020

\section{References}

1. Siegel RL, Miller KD, Jemal A. Cancer statistics, 2019. CA Cancer J Clin. 2019;69:7-34.

2. Mauro M, Rotundo G, Giancotti M. Effect of financial incentives on breast, cervical and colorectal cancer screening delivery rates: Results from a systematic literature review. Health Policy. 2019;123(12):1210-20.

3. Liontos M, Kyriazoglou A, Dimitriadis I, Dimopoulos MA, Bamias A. Systemic therapy in cervical cancer: 30 years in review. Crit Rev Oncol Hematol. 2019:137:9-17.

4. Sakai M, Sohda M, Saito H, Kuriyama K, Yoshida T, Kumakura Y, Hara K, Yokobori T, Miyazaki T, Murata K, Noda SE, Nakano T, Kuwano H, Shirabe K. Docetaxel, cisplatin, and 5-fluorouracil combination chemoradiotherapy for patients with cervical esophageal cancer: a single-center retrospective study. Cancer Chemother Pharmacol. 2019;83:1121-6.

5. Barra F, Leone Roberti Maggiore U, Bogani G, A D, Signorelli M, Martinelli F, Chiappa V, Lorusso D, Raspagliesi F, Ferrero S. New prophylactics human papilloma virus (HPV) vaccines against cervical cancer. J Obstet Gynaecol. 2019;39:1-10.

6. Perrone AM, Bovicelli A, D'Andrilli G, Borghese G, Giordano A, De laco P. Cervical cancer in pregnancy: analysis of the literature and innovative approaches. J Cell Physiol. 2019;234:14975-90.

7. Skinner SR, Apter D, De Carvalho N, Harper DM, Konno R, Paavonen J, Romanowski B, Roteli-Martins C, Burlet N, Mihalyi A, Struyf F. Human papillomavirus (HPV)-16/18 AS04-adjuvanted vaccine for the prevention of cervical cancer and HPVrelated diseases. Expert Rev Vaccines. 2016;15:367-87.

8. Shafabakhsh R, Reiter RJ, Mirzaei H, Teymoordash SN, Asemi Z. Melatonin: a new inhibitor agent for cervical cancer treatment. J Cell Physiol. 2019;234:21670-82.

9. Yun CW, Kim HJ, Lee SH. Therapeutic application of diverse marine-derived natural products in cancer therapy. Anticancer Res. 2019;39:5261-84.

10. Kumar MS, Adki KM. Marine natural products for multi-targeted cancer treatment: a future insight. Biomed Pharmacother. 2018;105:233-45.

11. Yao M, Qi Y, Bi K, Wang X, Luo X, Che C. A precolumn derivatization high-performance liquid chromatographic method with improved sensitivity and specificity for the determination of astragaloside IV in Radix Astragali. J Chromatogr Sci. 2000;38:325-8

12. Li L, Hou X, Xu R, Liu C, Tu M. Research review on the pharmacological effects of astragaloside IV. Fundam Clin Pharmacol. 2017;31:17-36.

13. Wang $\mathrm{HL}$, Zhou QH, Xu MB, Zhou XL, Zheng GQ. Astragaloside IV for experimental focal cerebral ischemia: preclinical evidence and possible mechanisms. Oxidative Med Cell Longev. 2017;2017:8424326.

14. Jia L, Lv D, Zhang S, Wang Z, Zhou B. Astragaloside IV inhibits the progression of non-small cell lung cancer through the Akt/GSK-3ß/ß-catenin pathway. Oncol Res. 2019;27:503-8.

15. Zhang L, Zhou J, Qin X, Huang H, Nie C. Astragaloside IV inhibits the invasion and metastasis of SiHa cervical cancer cells via the TGF- $\beta 1$-mediated PI3K and MAPK pathways. Oncol Rep. 2019;41:2975-86. 
16. Sun P, Liu Y, Wang Q, Zhang B. Astragaloside IV inhibits human colorectal cancer cell growth. Front Biosci (Landmark Ed). 2019;24:597-606

17. Wang S, Mou J, Cui L, Wang X, Zhang Z. Astragaloside IV inhibits cell proliferation of colorectal cancer cell lines through down-regulation of B7-H3. Biomed Pharmacother. 2018;102:1037-44.

18. Xia C, He Z, Liang S, Chen R, Xu W, Yang J, Xiao G, Jiang S. Metformin combined with nelfinavir induces SIRT3/mROSdependent autophagy in human cervical cancer cells and xenograft in nude mice. Eur J Pharmacol. 2019;848:62-9.

19. Noto JM, Rose KL, Hachey AJ, Delgado AG, Romero-Gallo J, Wroblewski LE, Schneider BG, Shah SC, Cover TL, Wilson KT, Israel DA, Roa JC, Schey KL, Zavros Y, Piazuelo MB, Peek RM Jr. Carcinogenic helicobacter pylori strains selectively dysregulate the in vivo gastric proteome, which may be associated with stomach cancer progression. Mol Cell Proteomics. 2019;18:352-71.

20. Li F, Wang Y, Li Y, Yang H, Wang H. Quantitative analysis of the global proteome in peripheral blood mononuclear cells from patients with new-onset psoriasis. Proteomics. 2018;18:e1800003.

21. Sun BB, Maranville JC, Peters JE, Stacey D, Staley JR, Blackshaw J, Burgess S, Jiang T, Paige E, Surendran P, Oliver-Williams C, Kamat MA, Prins BP, Wilcox SK, Zimmerman ES, Chi A, Bansal N, Spain SL, Wood AM, Morrell NW, Bradley JR, Janjic N, Roberts DJ, Ouwehand WH, Todd JA, Soranzo N, Suhre K, Paul DS, Fox CS, Plenge RM, Danesh J, Runz H, Butterworth AS. Genomic atlas of the human plasma proteome. Nature. 2018;558:73-9.

22. Cuomo F, Altucci L, Cobellis G. Autophagy Function and Dysfunction: Potential Drugs as Anti-Cancer Therapy. Cancers (Basel). 2019;11(10).

23. Amaravadi RK, Kimmelman AC, Debnath J. Targeting autophagy in cancer: recent advances and future directions. Cancer Discov. 2019;9:1167-81.

24. Xu F, Cui WQ, Wei Y, Cui J, Qiu J, Hu LL, Gong WY, Dong JC, Liu BJ. Astragaloside IV inhibits lung cancer progression and metastasis by modulating macrophage polarization through AMPK signaling. J Exp Clin Cancer Res. 2018;37:207.

25. Zhang WJ, Hufnagl P, Binder BR, Wojta J. Antiinflammatory activity of astragaloside IV is mediated by inhibition of NFkappaB activation and adhesion molecule expression. Thromb Haemost. 2003;90:904-14.

26. Zheng Y, Dai Y, Liu W, Wang N, Cai Y, Wang S, Zhang F, Liu P, Chen Q, Wang Z. Astragaloside IV enhances taxol chemosensitivity of breast cancer via caveolin-1-targeting oxidant damage. J Cell Physiol. 2019;234:4277-90

27. Denny P, Feuermann M, Hill DP, Lovering RC, Plun-Favreau H, Roncaglia P. Exploring autophagy with gene ontology. Autophagy. 2018;14:419-36.

28. Athanasios A, Charalampos V, Vasileios T, Ashraf GM. Protein-protein interaction (PPI) network: recent advances in drug discovery. Curr Drug Metab. 2017;18:5-10.

29. Xu X, Liu T, Yang J, Chen L, Liu B, Wang L, Jin Q. The first whole-cell proteome- and lysine-acetylome-based comparison between Trichophyton rubrum conidial and mycelial stages. J Proteome Res. 2018;17:1436-51.

30. Rao PS, Endicott R, Mullins R, Rao US. A 6-week laboratory research rotation in pharmacogenomics: a model for preparing pharmacy students to practice precision medicine. Pharmacogenomics J. 2018;18:601-8.

31. Azhar A, Ahmad E, Zia Q, Rauf MA, Owais M, Ashraf GM. Recent advances in the development of novel protein scaffolds based therapeutics. Int J Biol Macromol. 2017;102:630-41 Lin JJ, Riely GJ, Shaw AT. Targeting ALK: precision medicine takes on drug resistance. Cancer Discov 2017:7:137-55.

32. Ali R, Mirza Z, Ashraf GM, Kamal MA, Ansari SA, Damanhouri GA, Abuzenadah AM, Chaudhary AG, Sheikh IA. New anticancer agents: recent developments in tumor therapy. Anticancer Res. 2012;32(7):2999-3005 Cheng F, Zhao J, Fooksa M, Zhao Z. a network-based drug repositioning infrastructure for precision cancer medicine through targeting significantly mutated genes in the human cancer genomes. J Am Med Inform Assoc 2016;23:681-91.

33. Lin JJ, Riely GJ, Shaw AT. Targeting ALK: Precision Medicine Takes on Drug Resistance. Cancer Discov. 2017;7:137-55.

34. Cheng F, Zhao J, Fooksa M, Zhao Z. A network-based drug repositioning infrastructure for precision cancer medicine through targeting significantly mutated genes in the human cancer genomes. J Am Med Inform Assoc. 2016;23:681-91.

35. Vincent F, Loria P, Pregel M, Stanton R, Kitching L, Nocka K, Doyonnas R, Steppan C, Gilbert A, Schroeter T, Peakman MC Developing predictive assays: the phenotypic screening "rule of 3". Sci Transl Med. 2015;7:293ps15.

36. Moulder R, Bhosale SD, Goodlett DR, Lahesmaa R. Analysis of the plasma proteome using iTRAQ and TMT-based isobaric labeling. Mass Spectrom Rev. 2018;37:583-606.

37. Evans C, Noirel J, Ow SY, Salim M, Pereira-Medrano AG, Couto N, Pandhal J, Smith D, Pham TK, Karunakaran E, Zou X, Biggs CA, Wright PC. An insight into iTRAQ: where do we stand now. Anal Bioanal Chem. 2012;404:1011-27.

38. Bourmaud A, Gallien S, Domon B. Parallel reaction monitoring using quadrupole-Orbitrap mass spectrometer: principle and applications. Proteomics. 2016;16:2146-59.

39. Rauniyar N. Parallel reaction monitoring: a targeted experiment performed using high resolution and high mass accuracy mass spectrometry. Int J Mol Sci. 2015;16:28566-81.

40. Christian F, Anthony DF, Vadrevu S, Riddell T, Day JP, McLeod R, Adams DR, Baillie GS, Houslay MD. p62 (SQSTM1) and cyclic AMP phosphodiesterase-4A4 (PDE4A4) locate to a novel, reversible protein aggregate with links to autophagy and proteasome degradation pathways. Cell Signal. 2010;22:1576-96.

41. Morita T, Hayashi K. Tumor progression is mediated by Thymosin- $\beta 4$ through a TGF $\beta / M R T F$ signaling axis. Mol Cancer Res. 2018;16:880-93.

42. Scharlau D, Borowicki A, Habermann N, Hofmann T, Klenow S, Miene C, Munjal U, Stein K, Glei M. Mechanisms of primary cancer prevention by butyrate and other products formed during gut flora-mediated fermentation of dietary fibre. Mutat Res. 2009;682:39-53.

43. Kreutz D, Sinthuvanich C, Bileck A, Janker L, Muqaku B, Slany A, Gerner C. Curcumin exerts its antitumor effects in a context dependent fashion. J Proteome. 2018;182:65-72.

44. Xi T, Zhang G. Integrated analysis of tumor differentiation genes in pancreatic adenocarcinoma. PLoS One. 2018;13: e0193427.

\section{Publisher's Note}

Springer Nature remains neutral with regard to jurisdictional claims in published maps and institutional affiliations. 